\title{
Modelling travel and charging patterns of plug-in electric vehicles
}

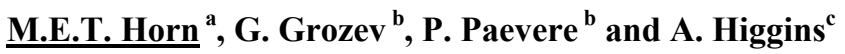 \\ a. CSIRO Computational Informatics, North Ryde, NSW. \\ b. CSIRO Ecosystem Sciences, Highett, Victoria. \\ c. CSIRO Ecosystem Sciences, Dutton Park, Queensland. \\ Email:mark.horn@csiro.au
}

\begin{abstract}
Plug-in electric vehicle (EV) technologies are now being offered in various forms by many of the major car manufacturers. These technologies offer several potential benefits, including energy security, utilization of EV batteries as load-balancing devices in electricity grids, and reduction in greenhouse gas emissions. The authors have developed an integrated framework for assessing these opportunities, through a sequence of models describing patterns of vehicle usage and battery recharge. The approach is disaggregated both in space and in time, in order to provide a sound operational basis for impact assessment.

The relevant patterns of vehicle usage are concerned with distance travelled during off-driveway periods (ODPs), and ODP departure and arrival times. In the modelling approach developed by the authors, these patterns are derived from travel surveys carried out by state Government agencies, where survey data is available; elsewhere, they are approximated using information from comparable areas.

The vehicle usage estimates are combined with estimates of the projected number of EVs in each Statistical Local Area to obtain profiles of total charging energy, which in turn are utilized by a suite of algorithms that model battery recharge/discharge processes. The processes modelled by these algorithms include peak and off-peak charging, and off-peak charging combined with vehicle-to-house discharging to support the grid at times of peak electricity consumption. The paper presents the modelling framework and methodology developed by the authors, and summarizes experience and results from a study of Townsville, Queensland.
\end{abstract}

Keywords: Travel behaviour; accessibility; electric vehicle; electric vehicle charging, energy demand 
Horn et al., Modelling travel and charging patterns of plug-in electric vehicles

\section{INTRODUCTION}

Many major vehicle manufacturers have developed plug-in electric vehicles (EV). While initial expectations for widespread adoption of EVs have not yet materialized, the technologies may yield substantial benefits. These include reduced dependence on depleting oil supplies; reduced emissions of greenhouse gas and other pollutants; and the ability to balance loads on the electricity grid through the use of EV batteries as distributed storage devices (e.g. charging the batteries at times when demand is low, and discharging when demand is high).

$\mathrm{EV}$ and other vehicle types are defined below.

BEV: A Battery Electric Vehicles is powered entirely by batteries charged from the grid.

HEV: A Hybrid Electric Vehicle is powered by batteries combined with an internal combustion engine.

PHEV: A Plug-in Hybrid Electric Vehicle is a HEV with batteries that can be charged from the grid

ICE: The Internal Combustion Engine is the main alternative to the various EV technologies.

The research described in this paper is concerned with assessing the potential impacts of a shift to EV usage on electrical distribution network loadings and operations, including opportunities for using EV batteries as network management components. The authors and their colleagues have been engaged in this research through a series of projects, of which the most recent is an analysis of energy demand and grid impacts in the Townsville Local Government Area (LGA), carried out by CSIRO for Ergon Energy. It is this Townsville study on which the present paper is based (Grozev et al., 2013).

Impact assessment entails a number of tasks, including formulating algorithms for network usage of EV batteries; projecting peak loads, quantifying the costs and benefits of emerging charging technologies, and assessing policies and pricing incentives related to EV purchasing and charging. For these purposes the authors and their colleagues have developed a modelling framework whose main components are concerned with EV uptake, EV usage patterns, household energy demand, and distribution network operation.

Other recent research has studied EV impacts in a variety of contexts, including power quality; energy efficiency and conservation; electricity demand patterns; greenhouse gas emissions; and the service life of distribution components. In a recent review of research on PHEV impacts, Green et al. (2011) emphasize the importance of an integrated modelling approach embracing a combination of driving patterns, charging characteristics, charging time and EV penetration. Another main issue is highlighted by Acha and van Dam (2013), who argue that a plausible determination of optimal charging EV profiles and associated grid impacts requires a fine-grained representation of spatial and temporal mobility patterns.

The research reported here addresses some of the critical issues referred to above, and represents a novel approach in its integration of a diverse range of models, and in its disaggregation of model elements both in space and in time. A disaggregated view is highly desirable, firstly in order to obtain a realistic view of the variability in behavioral patterns that may arise through combinations of demographic and geographic circumstances; and second, to enable the application of the modelling results to power systems planning at a local level. The modelling generally covers both PHEVs and BEVs, with some limitations in the detailed treatment of travel behaviour (see Section 2.2 below).

The analysis has been carried out using data defined under the Australian Statistical Geography Standard (ASGS), the successor to the former Australian Standard Geographical Classification (ASGC) (ABS-ASGS 2013). Where necessary, data collected under ASGC has been mapped to ASGS spatial categories, of which the most important for present purposes are as follows.

SA1: An SA1 is the smallest areal unit for which Census data is published (similar to CCD in ASGC). In urban areas there are around 200 households per SA1.

SA2: An SA2 is a composite of SA1s, and corresponds in urban areas to one or more suburbs (similar to SLA in ASGC).

\subsection{Overview of modelling framework}

The modelling framework developed by the authors has the following major elements (see Figure 1): 
Horn et al., Modelling travel and charging patterns of plug-in electric vehicles

- EV Diffusion Model to estimate EV uptake at three- monthly time intervals, under varying financial and policy scenarios, at SA1 level.

- EV Travel Model to estimate driving distances and the periods when EVs are available for charging and discharging, at SA2 level.

- $\quad$ EV Charging \& Discharging Model to estimate hour-byhour network loading based on EV uptake and travel patterns, and residential demand, at SA1 level.

- $\quad$ Residential Demand Model, to estimate residential energy usage (hourly x 365 days), at SA1 level. This model provides the background setting for energy usage.

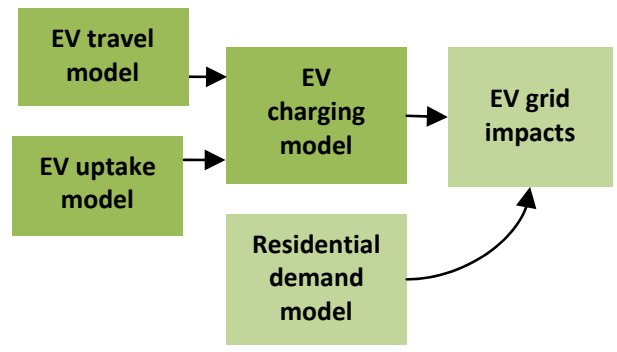

Figure 1. Modelling framework

The focus in this paper is on the integration of the first three of these models (highlighted in Figure 1) applying a conventional approach and on the demonstrated results in Section 3. Substantial technical effort has been required in order to obtain the desired level of granular detail in model outputs, as discussed in Section 1 and illustrated in Section 3.

\section{MODELLING COMPONENTS}

\subsection{Diffusion Model}

The task of the diffusion model is to estimate changes in composition of local vehicle fleets in terms of the various vehicles types (see Section 1). For the Townsville study, the evolution of these stocks was modelled at the SA1 level at three-month intervals, over a 30-year time-horizon. Three scenarios for promotion of EV uptake were investigated:

BASE: No rebates or incentives on EV purchases;

R5000: $\$ 5,000$ rebate on EV purchase price;

V2G500: \$500 per year incentive for vehicle-to-grid operation.

Modelling commences with the current stock of vehicles in each SA1, disaggregated by the owners' demographic groups. Forecasting for a given demographic group, SA1 unit and time-period commences with the propensity to make new purchases, given the age profile of the current stock, probability of product failure, disposition of each demographic group to novelty and early purchase, and availability of subsidies. For example, a high-income household may replace one of its vehicles sooner than a low-income household.

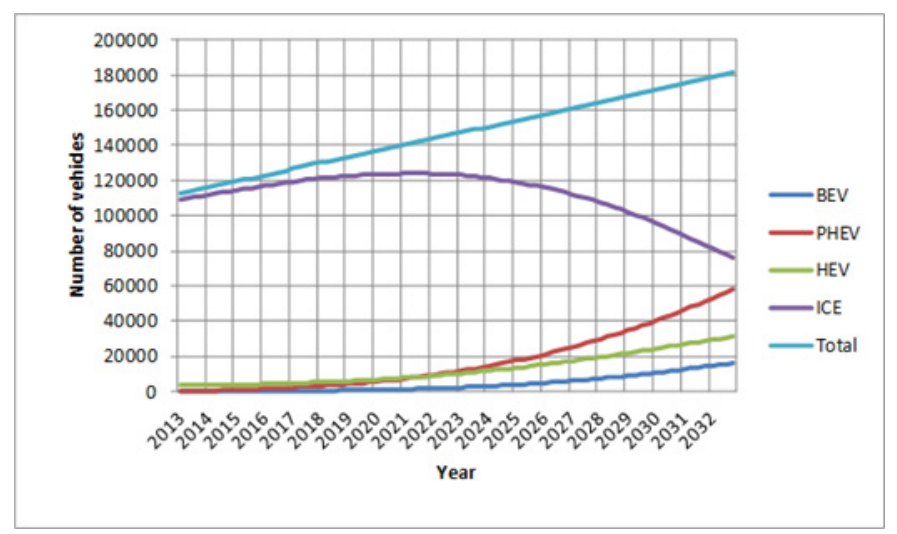

Figure 2. Aggregated vehicle stock projected for each vehicle option from 2013 to 2032 for the uptake scenario $=\mathrm{BASE}$

Once the number of households purchasing new vehicles has been estimated, the next decision involves the selection of vehicle types, and is modelled using a combination of choice-modelling theory and multicriteria analysis. The probability of a given consumer segment choosing a vehicle of a particular type, hence the impact on vehicle stocks, is obtained through the application of criteria pertaining to performance (driving range, top speed and comfort); annual operating costs; purchase price; household income; proximity to an urban area; demographic suitability (location, average age and persons per household, etc., by SA1); and familiarity (sales to date or stock of vehicle type relative to others). The demographic suitability score (range 0.0-1.0) was constructed from ABS and survey data by Gardner et al (2011), who identified key variables affecting consumers' decisions with respect to EV purchases. For eligible demographic groups, the R5000 and V2G500 schemes (see above) are modelled by adjusting relative criteria such as purchase price, operating cost, and familiarity (Higgins et al., 2012). An example of vehicle uptake for the study period and the BASE scenario is shown in Figure 2. 


\subsection{Travel Model}

Patterns of vehicle usage have significant impacts on the timing and duration of home charging of EVs, and hence on the charging profiles. The analysis of those patterns for Townsville has focused primarily on BEVs: PHEV recharge patterns are assumed to be similar to those for BEVs, scaled down to reflect the ICE-based recharge capability available in the case of PHEVs. An important assumption is that recharge takes place solely "on the driveway". This is feasible but excludes recharge at off-driveway "filling stations", using technologies such as fast recharge, battery swapping, or (for flow-cell batteries) electrolyte replacement. Although the commercial viability of such arrangements is uncertain, they are attractive (e.g. for rapid recharge and longer driving range), and may merit investigation in future analyses.

With home-based recharge, vehicle usage patterns at household level can be characterized in terms of OffDriveway Periods (ODPs), that is, the periods of each day when a vehicle is away from home. During an ODP a BEV expends energy, while recharge occurs after returning home and before the next ODP. That is,

- The distance travelled by a vehicle during an ODP determines the amount of battery recharge required after the BEV returns home; and

- The time when the vehicle returns home, and the duration of the period until subsequent departure, define the time-window during which recharge can take place.

The travel modelling task is to obtain ODP statistics, notably distributions of distance travelled, arrival and departure time, and ODP duration, on a local-area (i.e. SLA or SA2) basis. In previous studies for NSW and Victoria, these statistics were estimated from surveys of existing travel behaviour, using an activity-based approach (Horn, 2012). Similar survey data has been compiled for Townsville, but at the time of the work described here the State Government authorities declined to make them available.

An alternative approach was therefore developed, based on an adaptation of results from Newcastle NSW, a metropolitan region for which ODP statistics had previously been obtained and which is comparable in size and geographical situation to Townsville. The Townsville SLAs were assigned numeric scores according to their location within the region, and corresponding with a sub-regional classification (urban, suburban and outer-suburban) developed for Newcastle. An interpolations and rescaling was then performed for each SLA and required distribution, with further adjustments to the distance estimates using journey-to-work statistics obtained from the most recent Australian Census.

Although this approach has yielded plausible and robust results, it could be improve further in analytical terms. A systematic statistical methodology might be developed for use in future investigations where similar data shortfalls are present. Such an approach could also be valuable as a way of characterizing regions and localities in terms of travel behaviour, so as to obtain a more precise account of urban form than is available in current geographical theory (see Newman and Kenworthy 1995; Coeveringa and Schwanen 2006).

\subsection{EV charging and discharging model}

The EV charging and discharging model can simulate operations in any of the following charging modes:

- Demand charging (DEM). EVs recharge solely at their home base on arrival at home. Charging energy required is equal to the amount of energy used for the previous off-driveway travel.

- Off-peak charging (OP). EVs recharge solely at their home base with charging delayed until after midnight (12:00 am - 6:00 am) where 'on-driveway' time window permits.

- Off-peak charging with vehicle-to-house discharging (OPV2H): EVs recharge solely at their home base, daily, with charging delayed until after midnight where on-driveway time window permits. EVs can discharge energy to the house between 7:00 pm and 11:00 pm when present on the driveway, and subject to battery capacity and charging requirements.

Each charging mode has a set of parameters specifying time-slots when charging, peak charging, off-peak charging and vehicle-to-home discharging are permitted. Other charging mode parameters include charging and discharging powers, battery capacities, EV efficiency, priorities assigned to each time-slot, etc.

Given the outputs from the travel model and these parameters, the charge-discharge model uses simulation techniques to estimate the magnitude and timing of daily EV charging energy and power requirements, and of potential discharging events where relevant, for each charging mode. For each charging mode a charging profile for a single EV is constructed, which is derived from contributions of all possible trips (with given probabilities of travel from/to a given statistical area). The resulting charging profiles are multiplied by the 
projected numbers of BEV and PHEV in order to calculate the charging demand for a given statistical area and selected year into the future. For grid impact assessment this charging demand is compared with the residential electricity demand due to all other household uses.

\subsection{Residential electricity demand}

The residential demand model encompasses all domestic sources, including space heating and cooling, water heating, lighting and other appliances. Twelve prototype dwellings represent major components of the housing stock, including four dwelling types (detached, semi-detached, low-rise apartment/unit, high-rise apartment/unit) and three dwelling vintages (built 1991 or earlier, 1992-2006, 2007 or later). Three typical occupancy scenarios are modelled. Housing stock energy consumption is aggregated "bottom-up" from the end-use energy consumption of individual prototype houses (Ren et al., 2012).

\section{RESULTS}

The scenarios studied and major reporting levels for the Townsville study are illustrated in Figure 3: this provides the basis for identifying the cases illustrated in Section 3.2 below.

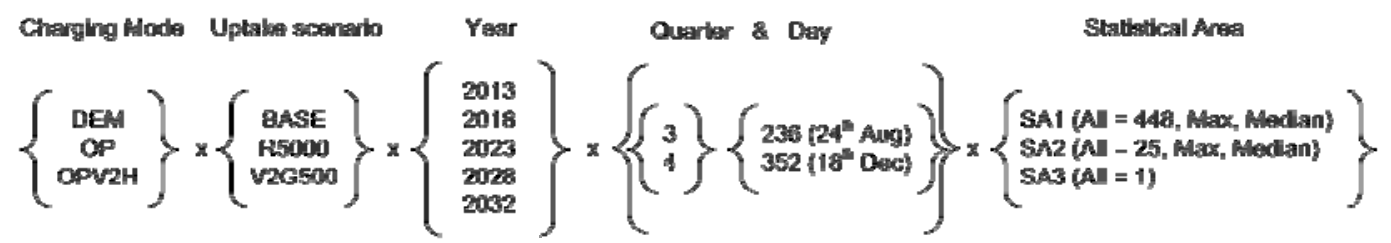

Figure 3. Scenarios and output levels

\subsection{Travel model results}

The distributions obtained from the EV Travel Model reflect differences in behavioral patterns across the region. This is illustrated by the ODP distance profiles (Figure 4); for example ODPs based in the central urban area (City, dotted red) are markedly shorter than in the semi-rural Northern Beaches-Pinnacles (purple line). Such differences reflect the Newcastle distributions, but are localized (e.g. through the use of journey-to-work data).

\subsection{Charging model results}

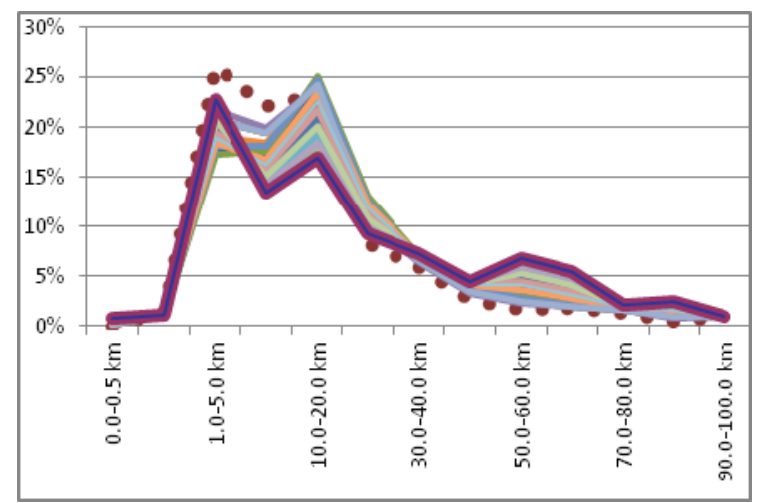

Figure 4. Off-driveway distances, by SLA

Figure 5 shows the three 24-hour charging profiles for each of the possible charging mode in a single SA1 area in the year 2028. The charging profiles are for the "maximum" SA1, which is the SA1 that has the highest value charging demand for a given uptake scenario (BASE) with comparison with all other SA1s under the same conditions. Demand charging has the smallest peak around 18:00-19:00 in the evening. Offpeak charging happens after midnight and before the morning and it creates higher peak at that time as the charging is restricted to only several hours in comparison with demand charging. Off-peak charging with vehicle-to-house discharging creates even higher peak after midnight (more than $450 \mathrm{~kW}$ for this particular SA1) as the batteries need to be charged additionally to compensate for the discharging to the house during the evening peak (see the negative peak close to 22:00).

Figure 6 shows charging in OPV2H mode across all SA1s in 2028. The highest peak (OPV2H) belongs to the same max SA1 with three charging profiles shown in Figure 5. Due to different uptake numbers of electric vehicles in different SA1s and due to different travel patterns the variation of the total charging demand for different statistical areas is significant. This Figure also demonstrates some limitations of the modeling approach, which generates very similar charging profiles for many statistical areas and for a given scenario.

Figure 7 shows the charging profiles for a single SAA1 at five-year intervals through the forecast period. The growth in the charging demand is explained with the increased numbers of electric vehicles as forecasted by the uptake model. The model assumes that the travel patterns will be kept the same throughout the study 
Horn et al., Modelling travel and charging patterns of plug-in electric vehicles

period and due to this reason it produces similar curves of the charging demand scaled differently for the selected years.

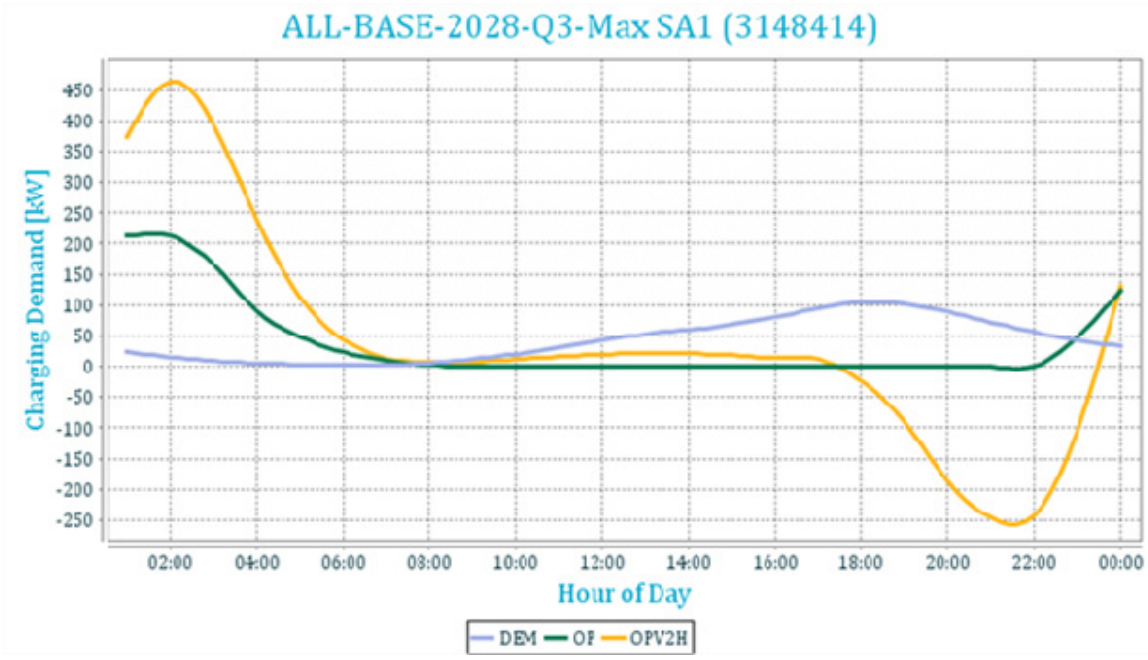

Figure 5. Charging profiles for all charging modes, one SA1, 2028

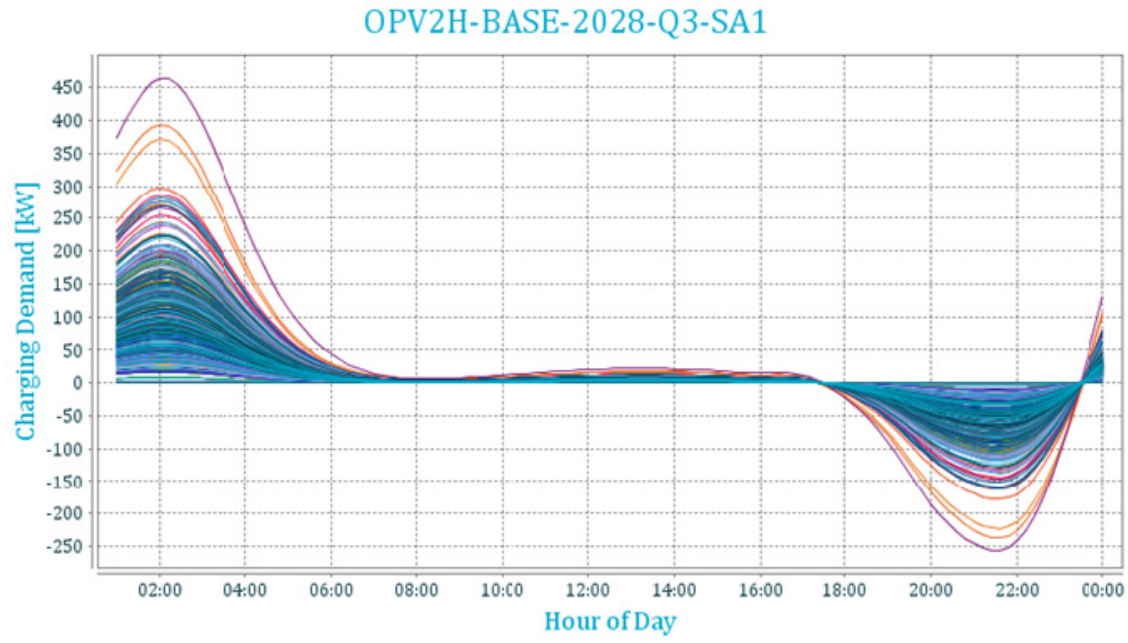

Figure 6. Off-peak vehicle-to-house charging at SA1 level, 2028

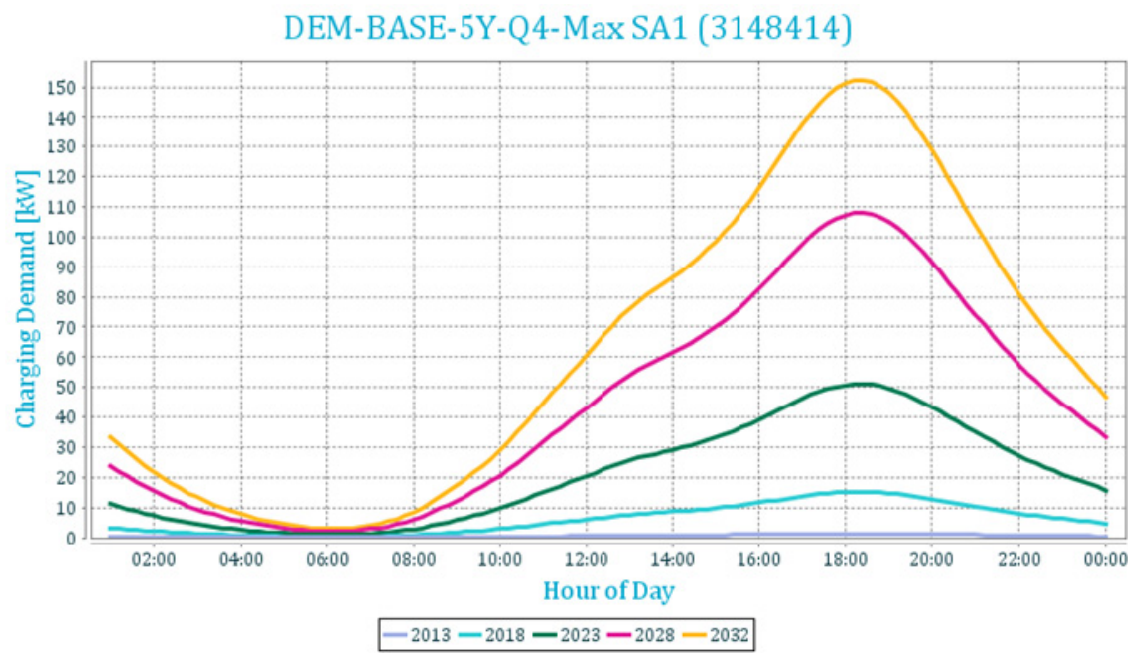

Figure 7. Demand profiles at SA1 for years 2013, 2018, 2023, 2028, 2032. 
Horn et al., Modelling travel and charging patterns of plug-in electric vehicles

\section{DISCUSSION AND CONCLUSIONS}

Assessment of modelling results is a complex task in itself (Grozev et al, 2013). In summary, the modelling for Townsville has shown that charging requirements for EVs, even with substantial future uptake, are well within the capacity of the existing electricity distribution network. In some charging modes (OP and OPV2H), the charging patterns of BEVs and PHEVs have significant implications for network management; for example, OPV2H may create a new peak after midnight and could significantly reduce the evening peak. Time-of-use charging rules, control of charging infrastructure and new electricity price tariffs may be needed to shift EV charging demand to periods of lower demand. EV batteries can support the grid, can be charged by renewable energy such as solar PV and can provide energy during peak electricity demand periods (afternoon or evening peak, depending on season and location).

Integrated spatial and temporal modelling is a key towards understanding of grid impact due to EV charging, and requires good models and data for other electrical loads at household/residential level. The analysis described here has demonstrated the feasibility and credibility of a disaggregated approach, although scope remains for improvement of the models described here (especially for travel patterns, household energy, and EV performance). In substantive terms, the scenario analyses have shown that with EV penetration levels estimated to 2032 by the diffusion model, the increase in peak electrical loads is only several percentage points and is manageable, given rules and electricity price tariffs that encourage off-peak charging. Off-peak charging combined with vehicle-to-grid discharging provides widespread benefits across time and space.

\section{ACKNOWLEDGMENTS}

This research was co-funded by Ergon Energy and the CSIRO Energy Transformed Flagship. The authors acknowledge the contributions of their CSIRO colleagues Dr. Zhengen Ren, Mr. Stephen Egan and Ms Julia Anticev, and Dr. Stephen Garner of Ergon Energy.

\section{REFERENCES}

ABS-ASGS (2013). ASGS Fact sheets. Available at http://www .abs.gov.au/websitedbs/D3310114.nsf/home/ASGS+Fact+Sheets

Acha, S. and van Dam, K.H. (2013). Modelling electric vehicle mobility in energy service networks. Chapter 7 in Modelling Distributed Energy Resources in Energy Service Networks. Available at http://digital-library.theiet.org/content/books/rn/pbrn016e

Coeveringa, P. van de and Schwanen, T. (2006). Re-evaluating the impact of urban form on travel patterns in Europe and North-America. Transport Policy 13(3), 229-239.

Gardner, J., Quezada, G., Paevere, P. (2011). Social study on attitudes, drivers and barriers to the uptake of electric vehicles. CSIRO Australia, Report EP113815.

Green II, R.C., Wang, L., Alam, M. (2011). The impact of plug-in hybrid electric vehicles on distribution networks: a review and outlook. Renewable and Sustainable Energy Reviews, 15, 544-553.

Grozev, G.V., Paevere, P., Higgins, A.J., Egan, S., Ren, Z., Horn, M.E.T., Anticev, J., Garner, S., Bloom, W. (2013). Modelling of electric vehicle charging patterns and impacts on peak electrical demand in Townsville, Queensland. CSIRO Australia, Report EP1310037.

Higgins, A.J., Paevere, P., Gardner, J., Quezada, G. (2012). Combining choice modelling and multi-criteria analysis for technology diffusion: an application to the uptake of electric vehicles. Technological Forecasting and Social Change, 79, 1399-1412.

Horn, M.E.T. (2012). Electric Driveway project: modelling of BEV travel for Victoria and other Australian states. CSIRO Report EP124967.

Newman, P.W.G. and Kenworthy, J.R. (1995). The land use - transport connection: an overview. Land Use Policy 13(1), 1-22.

Ren, Z., Paevere, P., and McNamara, C. (2012). A local-community-level, physically-based model of enduse energy consumption by Australian housing stock. Energy Policy, 49, 586-596. 